\title{
Analytical modeling and numerical simulation of heat transfer in a skin evaporator
}

\author{
Luka Lorbek*, Primož Poredoš, Andrej Kitanovski, Alojz Poredoš \\ Laboratory for Refrigeration and District Energy, University of Ljubljana, Faculty of Mechanical Engineering, Ljubljana, Slovenia
}

\section{A R T I C L E I N F O}

\section{Article history:}

Received 5 October 2017

Revised 26 January 2018

Accepted 31 January 2018

Available online 6 February 2018

\section{Keywords:}

Heat transfer

Household refrigerator

Analytical modeling

Skin evaporator

Numerical simulation

\begin{abstract}
A B S T R A C T
This paper presents a one-dimensional, steady-state, analytical and a two-dimensional, steady-state, numerical approach to describe heat transfer in the complex solid structure of a skin evaporator used in household refrigerators. Both models are based on a specific form of the energy equation (the heat diffusion equation), which is solved to obtain the temperature distributions and heat flows within the different parts of the structure. Heat transfer is limited by a number of thermal resistances, such as the inner plastic wall of the refrigerator, the aluminum plate, the foil, the tube of the evaporator, and the air gaps. The insulation that prevents heat flowing into the evaporator from the surroundings is also taken into consideration. A comparison of the models showed that the root-mean-square deviation of the calculated temperatures ranged from $0.34 \mathrm{~K}$ to $0.60 \mathrm{~K}$ and that the area-weighted discrepancy of the calculated heat flows was $4.6 \%$. Experimental validation of the model showed that the calculated and measured evaporator capacities were within $\pm 12 \%$. This demonstrates that several simplifications to the analytical model can be made, so drastically reducing the complexity of modeling a skin evaporator's structure, while retaining sufficient accuracy. These simplifications include reducing the heat transfer to one-dimensional heat conduction in the individual parts of the structure, neglecting the heat flux through the aluminumair-gap contact, and linearizing the aluminum foil.
\end{abstract}

(c) 2018 Elsevier Ltd and IIR. All rights reserved.

\section{Modélisation analytique et simulation numérique du transfert de chaleur dans un évaporateur à surface}

Mots-clés: Transfert de chaleur; Réfrigérateur domestique; Modélisation analytique; évaporateur à surface; Simulation numérique

\section{Introduction}

Household refrigerators use various types of evaporators, such as roll-bond (Hermes et al., 2008) or fin-tube (Lee et al., 2002) evaporators. In some refrigerators, however, a different type of evaporator, called a skin evaporator, has been introduced. This is a simple heat exchanger composed of (1) a bent tube, in which evaporation of the refrigerant takes place, (2) an aluminum plate, which serves as the structural support and to increase the heat transfer area, and (3) an aluminum foil, which holds the tube in place, and like the plate, increases the heat transfer area. The geometry is

Abbreviations: AG, air gap; CV, control volume.

* Corresponding author.

E-mail address: luka.lorbek@fs.uni-lj.si (L. Lorbek). similar to hot-wall or skin condensers (Rebora and Tagliafico, 1997; Bansal and Chin, 2002; Gupta and Gopal, 2008; Colombo et al., 2016). Their main advantages are low production costs, compared to other types of evaporator, and a slim profile. This makes them suitable for use in household refrigerators, where they can be positioned at the back or the sides of the refrigerated compartment. An example of a skin evaporator is shown in Fig. 1.

Despite having a simple production process, the elements of a skin evaporator form a complex structure. This presents a major challenge when it comes to describing the heat transfer within the structure. While the tube and plate have simple geometries, for which analytical solutions are well known, the foil that is pressed against the tube and the plate has a unique shape. This means that, because of the limits of the production process, the foil does not fit completely against the tube and the plate, which means that 


\begin{tabular}{|ll|}
\hline \multicolumn{2}{|c|}{ Nomenclature } \\
A & surface area $\left(\mathrm{m}^{2}\right)$ \\
C & integration constant $(/)$ \\
$c_{p}$ & specific heat at constant pressure $(\mathrm{J} / \mathrm{kg} \mathrm{K})$ \\
$h$ & heat transfer coefficient $\left(\mathrm{W} / \mathrm{m}^{2} \mathrm{~K}\right)$ \\
$H$ & insulation thickness in the $y$ direction $(\mathrm{m})$ \\
$k$ & thermal conductivity $(\mathrm{W} / \mathrm{m} \mathrm{K})$ \\
$q$ & heat flux $\left(\mathrm{W} / \mathrm{m}^{2}\right)$ \\
$Q$ & heat flow $(\mathrm{W})$ \\
$\mathrm{RMSD}$ & root-mean-square deviation \\
$S$ & cross-sectional area $\left(\mathrm{m}^{2}\right)$ \\
$T$ & temperature $(\mathrm{K})$ \\
$t$ & time $(\mathrm{s})$ \\
$U$ & overall heat transfer coefficient $\left(\mathrm{W} / \mathrm{m}^{2} \mathrm{~K}\right)$ \\
$w$ & width $(\mathrm{m})$ \\
Greek & letters \\
$\delta$ & thickness $(\mathrm{m})$ \\
$\rho$ & density $\left(\mathrm{kg} / \mathrm{m}^{3}\right)$ \\
Subscripts \\
a & air \\
C & outer tube wall \\
$\mathrm{F}$ & foil \\
$i$ & inner \\
$\mathrm{INS}$ & insulation \\
$o$ & outer \\
$\mathrm{PL}$ & plate \\
$r$ & refrigerant \\
$\mathrm{T}$ & tube \\
$\mathrm{V}$ & per unit volume \\
$\mathrm{W}$ & plastic wall \\
& \\
\hline
\end{tabular}

air gaps are formed in the structure. Furthermore, because of the presence of a contact resistance, the contact between the tube and the plate must also be considered. A scheme of the evaporator's cross-section is shown in Fig. 2, left, and a scheme of the hot-wall condenser is shown in Fig. 2, right.

A study of the literature in this field has shown that there are only a small number of studies covering heat transfer inside a skin evaporator with this kind of geometry, as presented in Fig. 1. There are, however, a handful of publications that relate to heat transfer in so-called hot-wall or skin condensers, which have a similar geometry to the skin evaporator.

One of the first attempts to analyze a hot-wall condenser and evaporator in a chest freezer for household applications was made by Rebora and Tagliafico (1997). They conducted a sensitivity study in order to achieve the best operating parameters for the freezer, compared to the usual refrigerator. The hot-wall condenser and evaporator of this freezer had the following geometry: a wall, composed of two adjacent plates that were placed on the outer/ambient and the refrigerated sides of the freezer. The gap between the plates was filled with insulation. On each inner side of the plate, a tube was in direct contact with the plate. The parameters that were studied were as follows: the total insulation thickness between both plates, the plate thickness, the tube diameter and the thermal contact resistance between the tube and the plate. To evaluate the freezer's performance a simplified, steady-state, 2D analysis involving a finite-element approach was performed. It was found that the largest factor influencing the freezer's performance was the insulation thickness. In addition, the heat transfer between the refrigerant and the ambient, as well as the freezer compartment, was influenced by the plate thickness and the thermal contact resistances between the tubes and the plates.

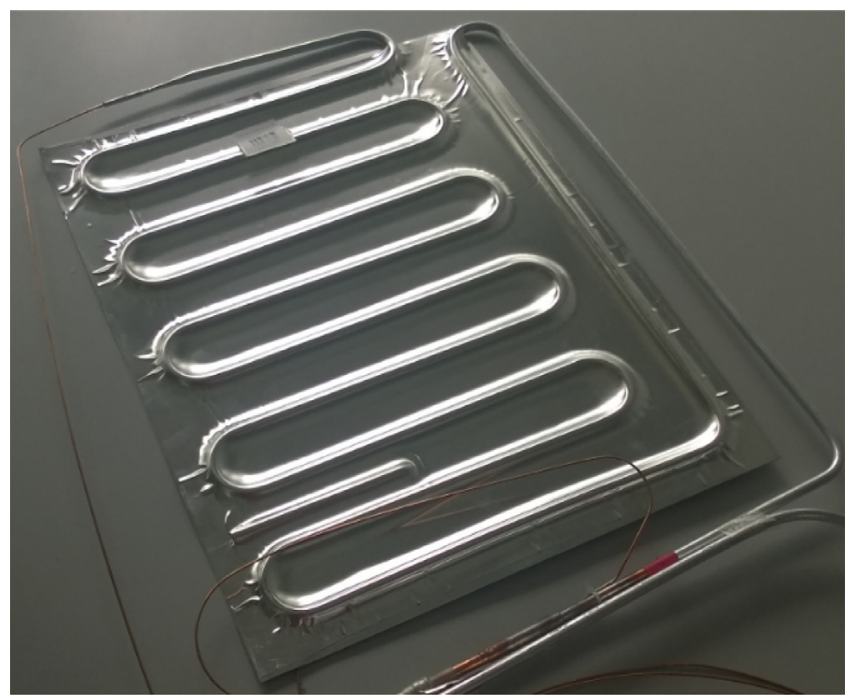

Fig. 1. Example of a skin evaporator.

Bansal and Chin (2002) conducted a study in which they compared their experimental data with the results of an analytical model of a hot-wall condenser. This condenser consisted of a steel tube that was in direct contact with the inner surface of the iron plate on the side walls of a refrigerator. An adhesive aluminum foil was also installed, which fixated the tube to the plate, but the model did not take into account the foil. Experiments were performed on a refrigerator using the R134a refrigerant. The simulation model employed a finite-element and variable-conductance approach. They found that the modeled condenser capacity was over-predicted by around $10 \%$ compared to the experimentally obtained results. The main reason for the disagreement was believed to be the heat transfer from the condenser to the refrigerator compartment. A similar study, which involved analytical modeling of a hot-wall condenser, but with the inclusion of the aluminum foil, was conducted by Gupta and Gopal (2008). The geometry of the condenser was the same as in the previous work (Bansal and Chin, 2002). The condenser tube was divided into elemental units, with each unit comprising the following basic elements: adhesive aluminum foil, tube and metal plate. The model considered an aluminum foil as a fin. The results showed that the modeled condenser capacity was over-predicted by around $2 \%$ compared to the experimental data, performed in (Bansal and Chin, 2002). It was also found that the aluminum foil has a significant impact on the heat transfer from the condenser to the ambient.

A study that compared the numerically and experimentally determined performance of a skin condenser was performed by Colombo et al. (2016). Both the aluminum foil and the steel plate were treated as independent fins. To validate the model they performed a series of experiments under different operating conditions. They reported that the difference between the calculated and experimental capacities was less than $2 \%$. A review of the literature showed that the heat transfer in hot-wall condensers has been studied in various ways, where most of the authors (Bansal and Chin, 2002; Gupta and Gopal, 2008; Colombo et al., 2016) treated parts of the condenser geometry as fins. Due to a similar structure the existing skin-condenser models could be modified to describe the skin evaporators; however, after our search of the existing literature, we found no studies that describe the heat transfer within the structure using only the heat diffusion equation. In the existing models more focus is given to the refrigerant's temperature distributions and the overall condenser capacity, while temperature dis- 

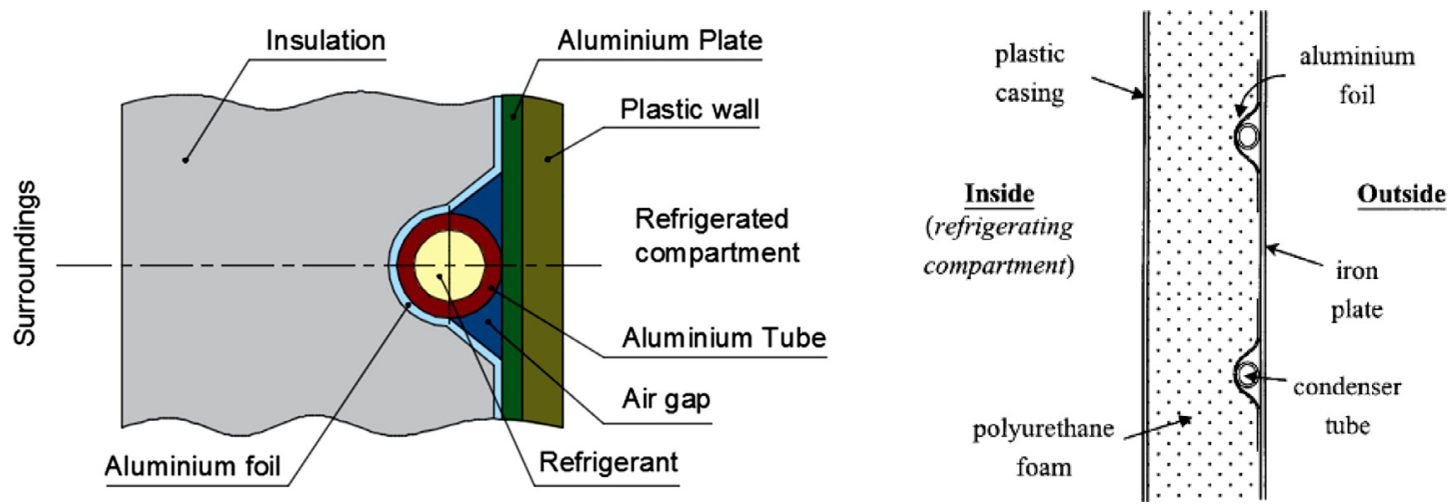

Fig. 2. (Left) Skin evaporator cross-section, (Right) hot-wall condenser cross-section (Bansal and Chin, 2002).

tributions and heat flows within the specific elements of the structure are not presented as extensively.

The purpose of this study was to present a simplified methodology for the analytical modeling of skin evaporators, based on the heat diffusion equation (a specific form of the energy equation), which describes the temperature distributions and heat flow in the entire structure of the evaporator. In order to compare the results, a numerical simulation in ANSYS Fluent and an experiment were performed, respectively. The study also shows the importance of including every element of the structure when modeling such heat exchangers as the aluminum parts of the evaporator present a relatively small thermal resistance. This means that heat transfer is substantially affected when other elements, such as the plastic wall of the refrigerator, are considered. The focus of the study was limited to the evaporator structure, in order to produce comprehensive results regarding the influence that each element has on the heat transfer within it.

\section{Analytical heat transfer model of the skin evaporator}

\subsection{Basic assumptions}

A set of assumptions relating to the approach of skin evaporator heat transfer modeling were made in order to reduce the complexity of the model:

- The heat transfer mechanism across the air gap was assumed to be based on heat diffusion (without convection). Neglecting the convective heat transfer was justified by determining the Rayleigh number, which was calculated for the air-gapaluminum-plate contact, considering the contact as a vertical wall. Convective heat transfer can be neglected if the Rayleigh number is less than some critical value. For a Benard configuration, which most closely resembles our case, the critical value is around 1708 (Manneville, 2006). The Rayleigh number in our case was of an order of magnitude of only 10 .

- The thermal contact between the tube and the plate was considered to represent an ideal line contact, i.e., adiabatic with no heat flow. The basis of this assumption was the calculation of an estimated ideal contact size between a cylinder and a wall, as presented by McGee et al. (1985). In the evaporator's production process a pressure of around $80 \mathrm{bar}$ is used when compressing the tube and foil to the plate. According to the model of McGee et al. (1985), the resulting contact size is around $0.18 \mathrm{~mm}$. Because the aluminum foil is not rigid, a much smaller contact force, and consequently contact size, can be expected after the production process. The contact between the tube and the plate is also not ideal, which means that a contact resistance is present, decreasing the heat flow through this

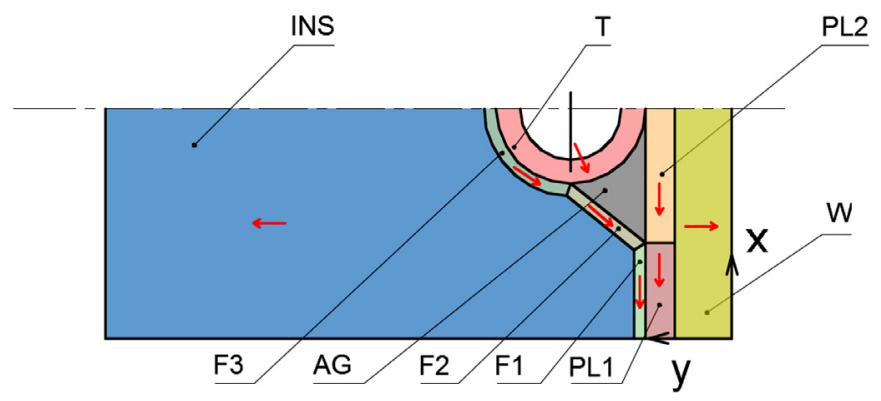

Fig. 3. Control volumes and assumed directions of the temperature gradients.

surface. However, it is important to note that the heat transfer through this contact is hard to describe, and that the manufacturing process plays a substantial role. The effect of this is visible in the number of different ways that similar models approached this problem.

- The evaporator tube was modeled as a straight tube.

- The heat fluxes were assumed to be constant along the width of the surfaces that they flow through.

\subsection{Governing equations}

The basis of the analytical model was the heat diffusion equation. For solid structures where there is no movement of the domain or diffusion through the surfaces the equation can be written as:

$\rho c_{p} \frac{\delta T}{\delta t}-\nabla \cdot(k \nabla T)=\dot{q}_{V}$

For steady-state conditions the first term, which describes the temperature change with time, can be neglected. The remaining terms describe the heat conduction through the solid and the heat generation:

$\nabla \cdot(k \nabla T)+\dot{q}_{V}=0$

For 2D problems this equation cannot be solved analytically, except for very simple geometries with the appropriate boundary conditions (Bergman et al., 2011). Because of this we divided our domain into control volumes for which the temperature gradient can be assumed to be one dimensional. The different colors in Fig. 3 show the specific control volumes and the red arrows mark the direction of the temperature gradient in that control volume.

Symmetry was assumed along the middle of the evaporator and only half of the structure was modeled. The foil was linearized so that it could be presented in a single coordinate system, yet 
the results were unaffected by this. For example, in the insulation a one-dimensional gradient can be assumed along its thickness ( $y$ axis), while in the foil a gradient can be assumed along its length ( $\mathrm{x}$ axis). With this assumption we can define a general governing equation for all control volumes, which can be described in the Cartesian coordinate system. The tube, for example, has to be described in radial coordinates. However, the temperature drop across the tube is expected to be very small, so only an energy balance will be presented for this control volume. Even though there is no heat generation present in the structure, the term describing heat generation will be kept as a source term, with which we will be able to describe the heat fluxes that flow into the control volumes perpendicular to the temperature gradient:

$\frac{d^{2} T}{d x^{2}}+\frac{\dot{q}}{k w}=0$

where $x$ represents the length of the control volume along the temperature gradient and $w$ represents the thickness of the control volume parallel to the temperature gradient. By integrating Eq. (2.3) twice for every corresponding control volume we can write the temperature-distribution functions in these control volumes. They are presented in Table A.1, in the appendix. In order to calculate the values of the temperature we must first resolve the heat fluxes flowing through the control-volume boundaries and then insert the boundary conditions to calculate the integration constants.

\subsection{Heat fluxes}

The heat fluxes were resolved independently by first estimating the heat fluxes from the surroundings and the refrigerated compartment to the refrigerant and then by applying energy-balance equations to the tube, plate, foil and air-gap control volumes. The heat flux from the surroundings can be estimated as:

$\dot{q}_{o}=U_{o}\left(T_{o}-T_{r}\right)=\frac{1}{\frac{1}{h_{o}}+\frac{\delta_{I N S}}{k_{I N S}}+\frac{\delta_{F}}{k_{F}}+\frac{\delta_{T}}{k_{T}}}\left(T_{o}-T_{r}\right)$

where the thermal resistance of the foil and tube are modeled as if they were straight walls. This simplification can be made because the terms representing the thermal resistance of the aluminum parts are almost negligible compared to the term representing the insulation. The heat flux from the refrigerated compartment can also be estimated as:

$\dot{q}_{i}=U_{i}\left(T_{i}-T_{r}\right)=\frac{1}{\frac{1}{h_{i}}+\frac{\delta_{W}}{k_{W}}+\frac{\delta_{P L}}{k_{P L}}+\frac{\delta_{T}}{k_{T}}}\left(T_{i}-T_{r}\right)$

where again the simplification of the thermal resistance can be justified based on the large ratio between the plastic wall and aluminum's thermal resistance.

The total energy balance can be written as:

$\dot{q}_{r} A_{r}=\dot{q}_{i} A_{i}+\dot{q}_{o} A_{o}$

Energy balances were then applied for the foil, plate, airgap, and tube control volumes. Because the surface area of the plastic-wall-refrigerated-compartment and plastic-wall-aluminumplate boundaries is the same the energy balance for the plastic wall can be omitted as the heat fluxes are equal. The heat flux from the interior $q_{\mathrm{i}}^{\mathrm{i}}$ is then taken as the heat flux through the plastic-wall-aluminum-plate boundary. The same was done for the insulation-aluminum-foil boundary, although a small error is introduced here as the surface areas of these boundaries are slightly different. This means that the heat flux entering the foil is slightly over-predicted. At this point one more equation is required to close the system of equations. To solve this, we assumed zero heat flux through the air-gap-aluminum-foil surface $\left(q_{\mathrm{a} 1}^{\cdot}=0 \mathrm{~W} / \mathrm{m}^{2}\right)$, which
Table 1

Boundary conditions.

\begin{tabular}{ll}
\hline Boundary & Boundary condition \\
\hline Surroundings-insulation & $T_{o}=303 \mathrm{~K}$ \\
& $h_{0}=10 \mathrm{~W} / \mathrm{m}^{2} \mathrm{~K}$ \\
Refrigerated compartment-plastic wall & $T_{i}=278 \mathrm{~K}$ \\
& $h_{i}=10 \mathrm{~W} / \mathrm{m}^{2} \mathrm{~K}$ \\
Interior tube wall-refrigerant & $T_{r}=258 \mathrm{~K}$ \\
Edges of symmetry & Adiabatic $(q=0)$ \\
\hline
\end{tabular}

we assumed would be close to zero, due to the temperature gradient in the air gap, which runs parallel to the mentioned surface.

Fig. 4 shows the control volumes, their surfaces and the heat fluxes that flow through them. Each color represents a specific control volume or boundary and the red arrows represent heat fluxes leaving or entering the control volumes. A sketch of the aluminum foil's linearization is also presented.

The energy-balance equations and Eq. (2.6) represent a system of equations that can be written in a matrix. To obtain the values of the heat fluxes the system of equations was solved in Matlab using the backslash operator. The required simulation time without post processing the results is $1.8 \mathrm{~s}$ on a $3.00-\mathrm{GHz}$ Intel $\mathrm{i} 7-5960 \mathrm{X}$ CPU.

$\left[\begin{array}{cccccccc}1 & -\frac{\boldsymbol{S}_{\mathrm{F} 1}}{\boldsymbol{A}_{\mathrm{F} 1}} & 0 & 0 & 0 & 0 & 0 & 0 \\ 0 & \frac{\boldsymbol{S}_{\mathrm{F} 1}}{\boldsymbol{A}_{\mathrm{F} 2}} & -\frac{\boldsymbol{S}_{\mathrm{F} 1}}{\boldsymbol{A}_{\mathrm{F} 2}} & 0 & 0 & 0 & 0 & 0 \\ 0 & 0 & \frac{\boldsymbol{S}_{\mathrm{F} 1}}{\boldsymbol{A}_{\mathrm{F} 3}} & -1 & 0 & 0 & 0 & 0 \\ 1 & 0 & 0 & 0 & \frac{\boldsymbol{S}_{\mathrm{PL} 1}}{\boldsymbol{A}_{\mathrm{F} 1}} & 0 & 0 & 0 \\ 0 & 0 & 0 & 0 & -\frac{\boldsymbol{S}_{\mathrm{PL} 1}}{\boldsymbol{A}_{\mathrm{PL} 2}} & 1 & 0 & 0 \\ 0 & 0 & 0 & 0 & 0 & 1 & \frac{\boldsymbol{A}_{\mathbf{C}}}{\boldsymbol{A}_{\mathbf{P} 2}} & 0 \\ 0 & 0 & 0 & 1 & 0 & 0 & 1 & -\frac{\boldsymbol{A}_{\boldsymbol{r}}}{\boldsymbol{A}_{\mathbf{C}}} \\ 0 & 0 & 0 & 0 & 0 & 0 & 0 & 1\end{array}\right]$

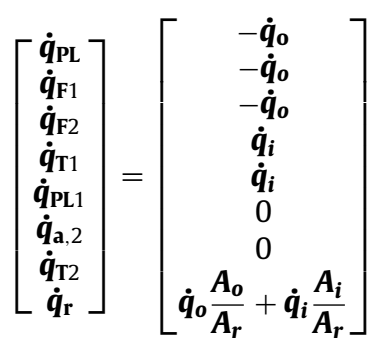

\subsection{Boundary conditions}

The applied boundary conditions are presented in Table 1. For the boundaries exposed to the refrigerated compartment and the surroundings we assumed a convective boundary condition. On the tube's interior we applied a constant-temperature boundary condition. The temperature of the surroundings $T_{0}$ is higher than the usual standard room temperature of around $25^{\circ} \mathrm{C}$, because this boundary is in the vicinity of the condenser.

The boundary conditions listed in Table 1 give sufficient information to run the numerical simulation. However, in order to be able to calculate the integration constants of the analytical model, a boundary condition has to be known for the primary unknown (temperature) and the secondary unknown (heat flux), for every control volume. Because of this, additional boundary conditions have to be assumed for certain control-volume boundaries. These are shown in Fig. 5, along with the boundary conditions listed in Table 1 (highlighted in the figure using bold). 


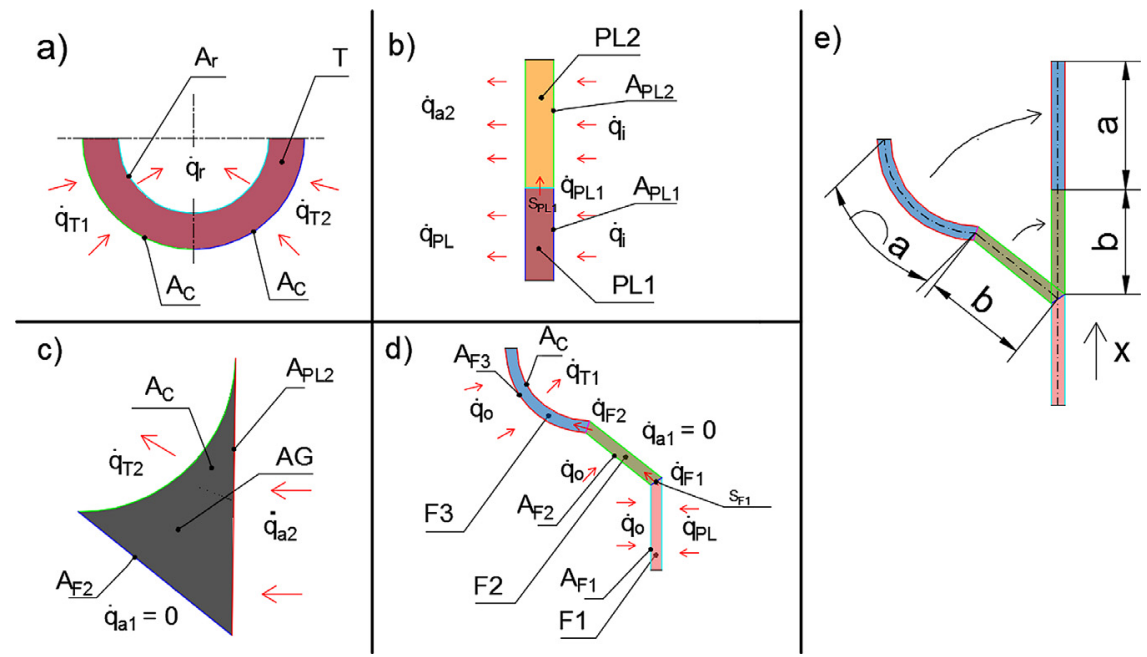

Fig. 4. (a) Tube CV, (b) Aluminum plate CV, (c) Air gap CV, (d) Aluminum foil CV, (e) Aluminum foil linearization.

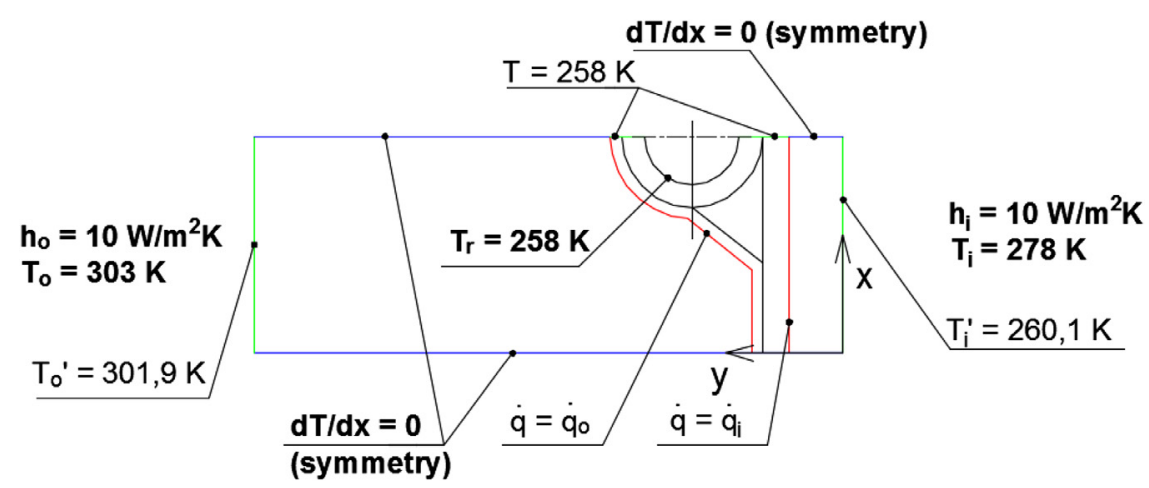

Fig. 5. Boundary conditions (bold) and additional boundary conditions for the analytical model.

The temperatures of the insulation $T_{0}$ ' and the plastic wall $T_{i}$, boundaries were determined using the known heat fluxes from Eqs. $(2.4,2.5)$, the constant free-stream temperature of the surroundings $\left(T_{0}\right)$ and the refrigerated $\left(T_{i}\right)$ compartment, and by only considering the convective thermal resistance $\left(h_{0}\right.$ and $h_{i}$ respectively) for the value of $U$.

The following thermal conductivities were considered:

- Aluminum parts: $202.4 \mathrm{~W} / \mathrm{mK}$

- Air: $0.023 \mathrm{~W} / \mathrm{mK}$

- Insulation: $0.024 \mathrm{~W} / \mathrm{mK}$

- Plastic wall: $0.19 \mathrm{~W} / \mathrm{mK}$

\section{Numerical heat transfer simulation of the skin evaporator}

For comparison a steady-state numerical simulation of the 2D heat transfer inside a skin evaporator was performed using ANSYS Fluent. An unstructured conformal computational mesh with around 7178 triangular and quadratic nodes was created in ANSYS Meshing. The foil was divided into $0.1 \mathrm{~mm}$-wide quadratic elements, with three elements along the thickness. The insulation was meshed with triangular elements that ranged in size from $0.1 \mathrm{~mm}$ at the foil contact to $2.85 \mathrm{~mm}$ at the edge. The air gap and tube were meshed with $0.1 \mathrm{~mm}$ - to $0.15 \mathrm{~mm}$-large triangular and quadratic elements. Because the mesh was conformal the width of the quadratic elements on the aluminum plate and plastic wall was automatically set to $0.1 \mathrm{~mm}$. Along the thickness of the plate there were 5 uniformly spaced divisions, and along the thickness of the wall there were 11 divisions with a bias factor of 5 , with the larger elements at the edge of the domain. The mesh quality was then checked with the skewness parameter. An equiangular cell is considered as an ideal case for a control-volume shape. The skewness parameter (ANSYS, Inc 2016) describes with a value from 0 to 1 how close a cell is to the ideal shape, with 0 being the ideal case. A rule of thumb (ANSYS, Inc 2016) is that no cells should have a skewness larger than 0.95 . Four cells with a skewness parameter of 0.94 represent the most skewed cells in our case. However, the average skewness of the mesh was less than 0.1 .

Similar to the presented analytical model, ANSYS Fluent uses the heat diffusion equation (ANSYS, Inc, 2016) as its governing differential equation for describing the temperature change in a solid in space or over time. Because the heat diffusion equation is solved numerically, using the finite-volume method, no simplification to $1 \mathrm{D}$ heat conduction is necessary. The interior parts of the skin evaporator's structure are therefore coupled with an interior boundary condition, which assumes equal temperature and heat flux on the interior boundary between two objects. The boundary conditions used in the simulation are presented in Table 1. Spatial discretization of the gradients was performed with the leastsquares cell-based method, as the result is expected to vary linearly. Without the convective term, as is the case for a stationary solid domain, velocity is no longer present in the heat diffusion equation. There is also no pressure term. Because the solution methods mostly deal with the coupling and discretization of pressure and velocity, the results turned out to be independent of the choice of solution methods. The absolute residual criteria was set to $10^{-9}$. Simulations were also performed on three meshes of variable grid density to ensure that the results are independent of the grid. The number of nodes on the coarse grid was 1232 and the 


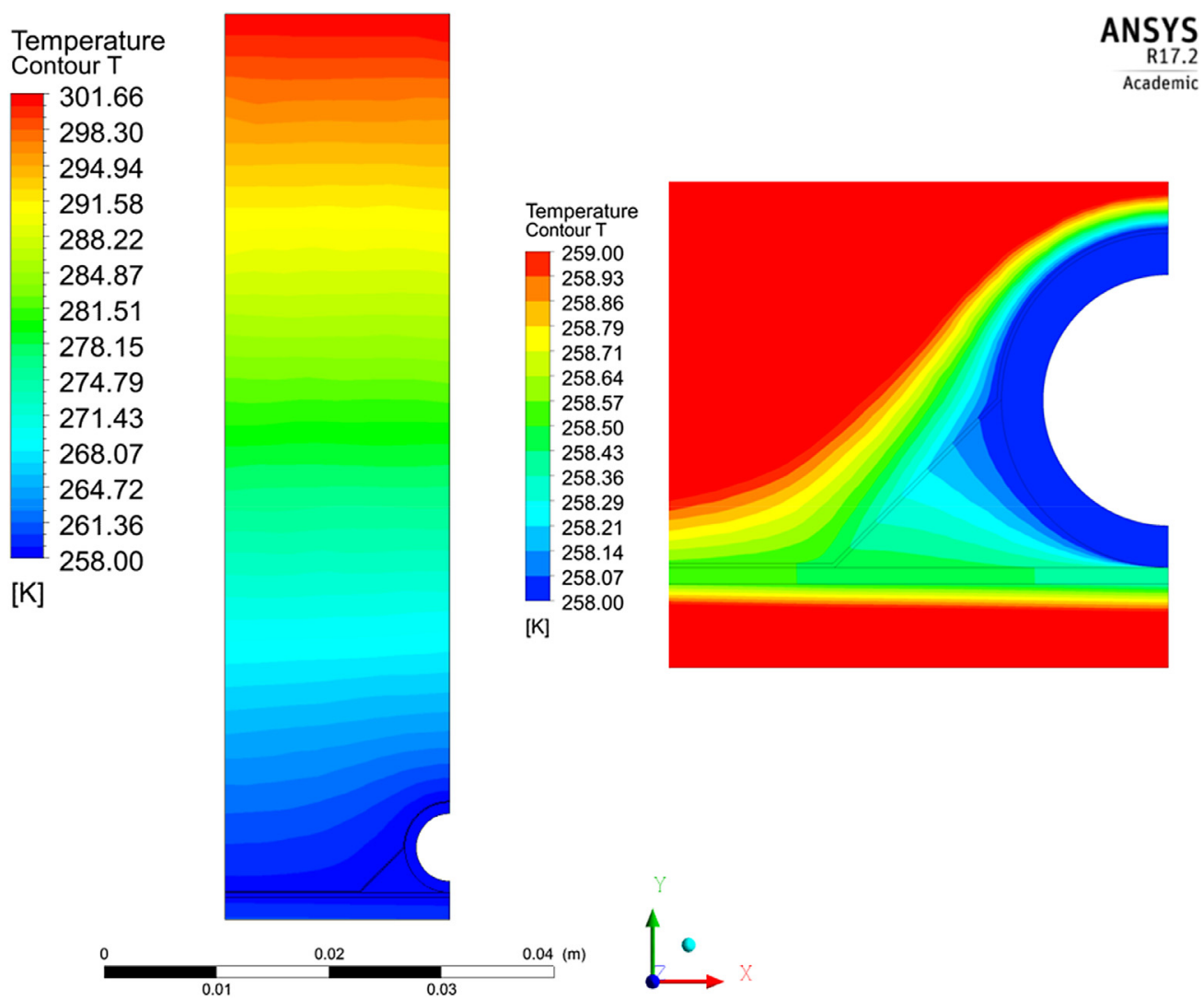

Fig. 6. (Left) Temperature contours of the modeled domain, (Right) detailed temperature contours from $258 \mathrm{~K}$ to $259 \mathrm{~K}$ around the air gap.

number of nodes on the fine grid was 49,970. Results between the different meshes varied by a maximum of $2.4 \%$, which shows that they are grid independent.

\section{Results}

\subsection{Temperature distributions}

The temperature distributions obtained with the numerical simulation in ANSYS Fluent are presented in Fig. 6. On the left, the temperature contours along the entire structure of the evaporator are shown. We can see that the temperature differences from the inner tube wall to the refrigerated compartment, or along the foil, are very small, as they are all within a margin of $4 \mathrm{~K}$. Because the insulation creates the highest temperature difference, the temperature resolution of the contours around the aluminum parts is too small. To solve this problem, we can only adjust the temperature range from $258 \mathrm{~K}$ to $259 \mathrm{~K}$ and focus on the area around the air gap (Fig. 6, right). It is clear that the temperature drop across the tube is negligible. Also visible are the temperature contours in the air gap, which run perpendicular to the air-gap-aluminum-foil contact (Fig. 6, right). Because there is almost no temperature gradient parallel to the air-gap-aluminum-foil contact, only a minimal heat flow through this surface is present. This shows that the assumption of zero heat flux through this contact in the analytical model is acceptable.

An interesting result is that the entire aluminum structure is within a margin of less than $1 \mathrm{~K}$. Three conclusions can be made on the basis of this observation:

- The small temperature difference can be attributed to the heat conduction along the aluminum foil, which shows the importance of including it in the calculation.

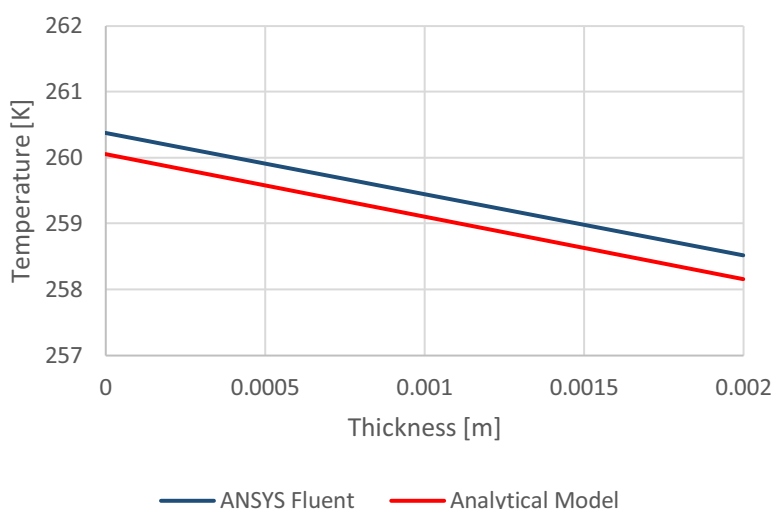

Fig. 7. Temperature distribution in the plastic wall.

- The effect of the air gap on heat transfer is not significant. While it is essential to include it in the calculation of the heat flows, the air gap itself does not present a significant thermal resistance, since a large amount of heat flux can be conducted around it along the aluminum foil.

- Besides serving as an extended surface, the aluminum plate has only a small temperature fluctuation along its width due to the high conductivity of the aluminum. This means that the heat flow from the refrigerated compartment is distributed evenly along the entire evaporator surface.

Additionally, we can see that thermal resistances based on convective heat transfer and conduction through the plastic wall represent the majority of the thermal resistances. The next paragraph discusses this in more detail.

Fig. 7 shows the temperature distributions in the plastic wall along the $y$ direction. The temperature distribution of the numeri- 


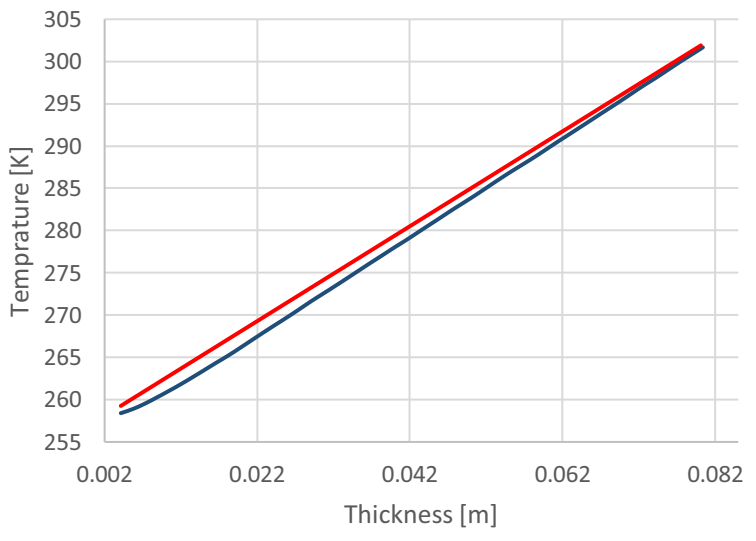

- ANSYS Fluent —-Analytical Model

Fig. 8. Temperature distribution in the insulation.

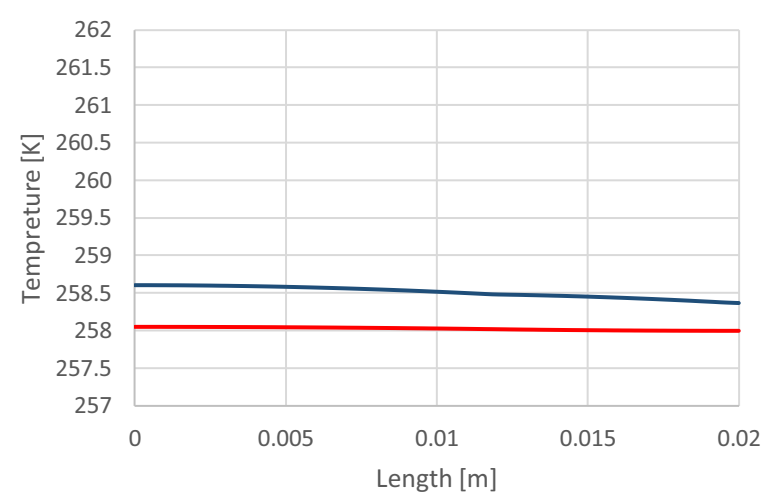

- ANSYS Fluent $\quad$ Analytical Model

Fig. 9. Temperature distribution in the aluminum plate.

cal solution is presented as an average of three temperature distributions along the $\mathrm{x}$ coordinate of the plastic wall (this statement is also valid for the temperature distributions in the insulation).

It is clear that the plastic wall represents a higher thermal resistance compared to the aluminum parts, because the temperature difference across the wall is around $2 \mathrm{~K}$. This is more than for the entire aluminum structure, which we described as being within a margin of less than $1 \mathrm{~K}$. The free-stream temperature in the convective boundary condition at the plastic-wall-refrigeratedcompartment boundary was set to $278 \mathrm{~K}$, but the edge of the plastic wall has a temperature of only around $260 \mathrm{~K}$. This means that there is an almost $18 \mathrm{~K}$ drop in the temperature across the boundary layer, due to a very small heat transfer coefficient. This agrees with the discoveries made by Bansal and Chin (2002) that convective heat transfer resistances represent more than $80 \%$ of all the thermal resistances in such a structure. The values of the temperature between the analytical and numerical models are comparable, with a root-mean-square deviation (RMSD) of $0.34 \mathrm{~K}$. The linear trend of the numerical solution agrees with the assumption used in the analytical model, that the heat conduction in the wall is one dimensional.

The temperature distributions along the y direction in the insulation are presented in Fig. 8. The RMSD between the models is $0.60 \mathrm{~K}$. Again, a one-dimensional heat conduction trend can be observed.

Fig. 9 presents the temperature distributions along the $\mathrm{x}$ direction of the aluminum plate. The RMSD between the models is $0.49 \mathrm{~K}$. The analytical model shows a negligible temperature differ-

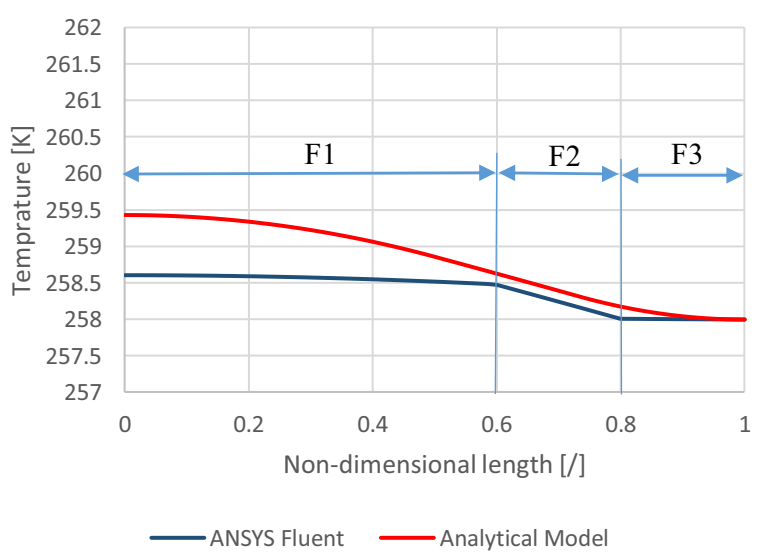

Fig. 10. Temperature distribution in the aluminum foil.

ence along the length of the plate and the numerical simulation suggests there is a minor temperature difference of around $0.3 \mathrm{~K}$. This shows how the aluminum plate evenly distributes the temperature, allowing the entire plate to be active in the heat transfer process.

Fig. 10 shows the temperature distribution along the length of the aluminum foil. Because we linearized the foil in the analytical model, the temperatures have to be presented along a nondimensional length from 0 to 1 in the positive $\mathrm{x}$ direction. There is a larger discrepancy in the control volume F1, compared to the other control volumes of the foil. We believe this can be directly connected to the discrepancy in the heat flux on the Al-plate-Alfoil boundary, shown in the next section in Table 2. The RMSD between the models, however, is still only $0.4 \mathrm{~K}$.

\subsection{Heat flows}

Table 2 presents the heat flows and their distribution in the evaporator structure. The total heat flow to the refrigerant is $7.8 \mathrm{~W}$ per $1 \mathrm{~m}$ length of the evaporator tube. The usual tube length in such evaporators is around $8 \mathrm{~m}$, which means that the total cooling capacity would be $62.4 \mathrm{~W}$. The heat flow from the surroundings, through the insulation, amounts to around $6.7 \%$ of the total heat flow to the refrigerant. This means that $6.7 \%$ of the heat flow does not contribute to cooling the refrigerated compartment. Of course this value is dependent on the thermal conductivity of the insulation and increases with a higher thermal conductivity. The distribution of heat flow through the structure also shows the importance of the aluminum foil. More than $40 \%$ of the heat flow that is conducted through the plastic wall and the aluminum plate is transferred to the aluminum foil. Heat flow from the aluminum plate to the foil is then conducted along the length of the foil to the aluminum tube. Similarly, heat flow from the air gap is also transferred to the aluminum tube. The numerical results also support our assumption that the heat flow between the air gap and the aluminum foil can be neglected. Because symmetry was assumed along the middle of the evaporator, the results for the heat flows in the boundary contacts in Table 2 are equal to half of the real values for the domain shown in Fig. 2.

The discrepancy between the numerical and analytical results ranges from $1.7 \%$ to $11.7 \%$. We believe a part of this discrepancy can be attributed to the assumption of constant heat flux along the length of our control volumes. This assumption is the least accurate in the case of the aluminum plate and the foil contact, where the temperature gradient along the length of the foil is not negligible. This agrees with our results as the discrepancy between the models for this contact is $10.8 \%$. Because the heat flow along the 
Table 2

Heat flows and their distribution in the modeled evaporator structure.

\begin{tabular}{|c|c|c|c|c|}
\hline Contact & Index & Heat flow (W)-Numerical simulation & Heat flow (W)-Analytical model & Discrepancy (\%) \\
\hline \multicolumn{5}{|l|}{ Boundary contacts } \\
\hline $\begin{array}{l}\text { Plastic wall-Refrigerated compartment } \\
\text { Tube interior-Refrigerant } \\
\text { Insulation-Surroundings } \\
\text { Residual* }\end{array}$ & $\begin{array}{l}Q_{\mathrm{i}} \\
Q_{\mathrm{r}} \\
Q_{0}^{\prime} \\
Q_{R}^{\prime}\end{array}$ & $\begin{array}{l}3.53 \\
3.69 \\
0.27 \\
0.11\end{array}$ & $\begin{array}{l}3.62 \\
3.88 \\
0.26 \\
1\end{array}$ & $\begin{array}{l}2.54 \\
4.88 \\
4.58 \\
\text { I }\end{array}$ \\
\hline \multicolumn{5}{|l|}{ Interior contacts } \\
\hline $\begin{array}{l}\text { Plastic wall-Al plate } \\
\text { Al plate-Al foil } \\
\text { Al plate-Air gap } \\
\text { Al foil-Air gap } \\
\text { Al foil-Insulation } \\
\text { Al foil-Tube } \\
\text { Air gap-Tube }\end{array}$ & $\begin{array}{l}Q_{\mathrm{i}}^{\prime} \\
Q_{\mathrm{PL}}^{\prime} \\
Q_{\mathrm{a} 2}^{\prime} \\
Q_{\mathrm{a} 1}^{\prime} \\
Q_{\mathrm{INS}}^{\prime} \\
Q_{\mathrm{T} 1}^{\prime} \\
Q_{\mathrm{T} 2}^{\prime}\end{array}$ & $\begin{array}{l}3.53 \\
1.58 \\
1.92 \\
0.01 \\
0.28 \\
1.79 \\
1.89\end{array}$ & $\begin{array}{l}3.62 \\
1.77 \\
1.85 \\
0 \\
0.31 \\
2.02 \\
1.85\end{array}$ & $\begin{array}{l}2.54 \\
10.78 \\
4.06 \\
/ \\
2.18 \\
11.67 \\
1.72\end{array}$ \\
\hline
\end{tabular}

* this is the loss of energy conservation due to various errors in the numerical simulation.

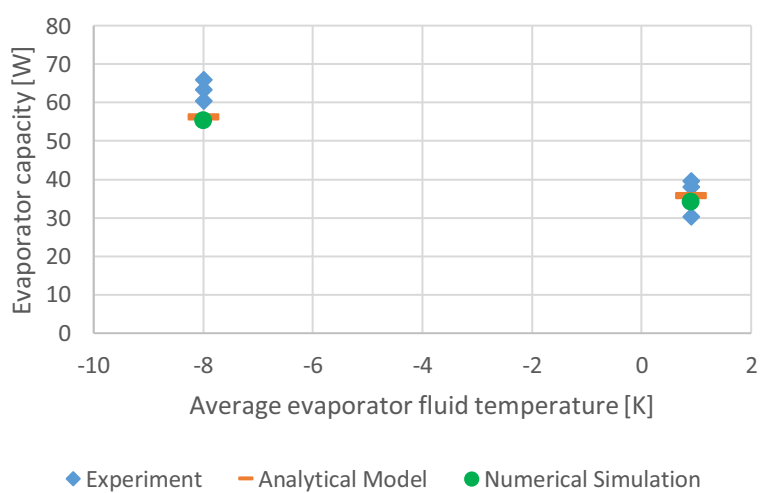

Fig. 11. Comparison of our experimental and model results.

foil conducts straight to the tube, we assume that the error at the foil-tube contact is directly related to the mentioned discrepancy. Some difference also lies in the way we determined the heat flows $q_{o}$ and $q_{i}^{*}$ from the refrigerated compartment and surroundings.

\subsection{Comparison with experimental results}

An experiment was performed on a bottom-mount refrigerator with a skin evaporator, which was stripped of the capillary tube and the compressor. The evaporator's inlet and outlet were connected to a thermostatic bath with an in-built pump, which circulated ethanol through the skin evaporator. The temperature on the evaporator inlet could be controlled to $\pm 0.1^{\circ} \mathrm{C}$ and 6 measurements were performed at inlet temperatures of $-10^{\circ} \mathrm{C}$ and $0{ }^{\circ} \mathrm{C}$. A high mass flow rate through the evaporator was set, so that the temperature change from the inlet and outlet would be as small as possible. In this way the experiment would be more comparable to the models that have a fixed-temperature boundary condition on the tube interior. K-type thermocouples were used, with an uncertainty of $\pm 0.1^{\circ} \mathrm{C}$. The evaporator's cooling capacity was determined by measuring the ethanol mass flow and the evaporator's inlet and outlet temperatures. The mass flow was determined by measuring the mass change with respect to time in a graduated cylinder. The weighing scale with an accuracy of $\pm 1 \mathrm{~g}$ was connected to a computer for real time mass measurements. The measurement lasted until a change of $400 \mathrm{~g}$ was achieved. While the accuracy of the instruments was sufficient, it is crucial to consider another factor. Both the density, and consequentially the mass flow are functions of temperature. Mass flow can thus only be measured correctly if the temperature does not change anymore, with respect to time. In order to minimize the measuring uncertainty as much as pos- sible, the flow was measured after the thermal conditions reached a steady state. The refrigerator compartment was held at a constant temperature by means of an electric heater and the test was performed in a controlled atmosphere chamber, with a constant ambient temperature of $25^{\circ} \mathrm{C}$. The evaporator's geometry was the same as the one used in the analytical model, but with a length of $8.5 \mathrm{~m}$ and an inner tube diameter of $7 \mathrm{~mm}$. In their study Laguerre and Flick (2004) presented an estimation of the heat transfer coefficient for the evaporator-refrigerated-compartment boundary in a household refrigerator. If the temperature of the refrigerator walls and the refrigerated air is assumed to be similar, the heat transfer coefficient can be written as the sum of the convective and radiative terms. According to their results the combined heat transfer coefficient would be around $7.1 \mathrm{~W} / \mathrm{m}^{2} \mathrm{~K}$. A constant value of $7.5 \mathrm{~W} / \mathrm{m}^{2} \mathrm{~K}$ was therefore assumed for the inner heat transfer coefficient when comparing the analytical and numerical results to the experiment. The results of the comparison are presented in Fig. 11.

The calculated results were found to be within $\pm 12 \%$ of the experimental results. However, it must be noted that the overall calculated evaporator capacity is very sensitive to the presumed heat transfer coefficients. This makes sense as the convective thermal resistance represents around $85 \%$ of the overall thermal resistances and even a very small change in the heat transfer coefficient has a great impact on the result.

\section{Conclusions}

This paper presents an analytical model for describing the heat transfer in a skin evaporator's structure, using basic heat transfer equations, and a numerical simulation to serve as a reference for the analytical model. The results between the model and the simulation were found to be in agreement, with the RMSD of the temperature ranging from 0.34 to $0.60 \mathrm{~K}$ in a domain with a temperature span of $45 \mathrm{~K}$. The average area-weighted discrepancy of the heat flow between the model and the simulation is $4.6 \%$. Based on these results we can conclude that the assumed simplifications in the analytical model do not lead to a significant difference compared to the numerical model. We believe that the most influential simplifications are the simplification to the one-dimensional heat conduction in the respective control volumes and the assumption of constant heat fluxes along the surfaces where there is a temperature gradient. These assumptions also present the largest constraints for the model. For example, it would not be applicable for cases that do not exhibit one-dimensional properties. However, since the geometry of such an evaporator is very specific, substantial modifications to the evaporator geometry would mean that it would lose the traits of a skin evaporator, making such modifications impractical. Other reasons that cause discrepancy between 
the models include the numerical and discretization errors in the numerical model. An experiment was also performed in order to validate the analytical model and numerical simulation. To achieve comparable results the measurements were made in a bottommount refrigerator whose structural elements were the same as those regarded in the model and simulation. A discrepancy of no more than $\pm 12 \%$ was observed.

The temperature drops across the control volumes, from the refrigerant to the refrigerated compartment, also show that the convective thermal resistance represents around $85 \%$ of the total resistance, the plastic wall around $10 \%$, and the air gap along with the aluminum parts (plate, tube, and foil) the other 5\%. From this we can conclude that the air gap does not represent a large thermal resistance. We believe that this is due to the aluminum foil, which conducts a considerable amount of heat around the air gap. Future work will involve a parametric study regarding the limits of the model, as it could determine when the simplification to onedimensional heat conduction would no longer be appropriate.

\section{Acknowledgments}

This work was financially supported by the Slovenian Research Agency as part of the Young Researcher PhD program.

\section{Appendix A}

Table A.1

Temperature distribution functions

\begin{tabular}{ll}
\hline Control volume & Temperature distribution equation \\
\hline Foil-F1 & $T_{F 1}(x)=-\left(\frac{\dot{q}_{P L}}{\lambda_{F} W_{F}}+\frac{\dot{q}_{o}}{\lambda_{F} W_{F}}\right) \frac{x^{2}}{2}+C_{1} x+C_{2}$ \\
Foil-F2 & $T_{F 2}(x)=-\left(\frac{\dot{q}_{o}}{\lambda_{F} W_{F}}\right) \frac{x^{2}}{2}+C_{3} x+C_{4}$ \\
Foil-F3 & $T_{F 3}(x)=-\left(\frac{q_{F 1}}{\lambda_{F} W_{F}}+\frac{\dot{q}_{o}}{\lambda_{F} W_{F}}\right) \frac{x^{2}}{2}+C_{5} x+C_{6}$ \\
Al plate-PL1 & $T_{P L 1}(x)=\left(\frac{q_{P L}}{\lambda_{P P} W_{P L}}-\frac{\dot{q}_{i}}{\lambda_{P L} w_{P L}}\right) \frac{x^{2}}{2}+C_{7} x+C_{8}$ \\
Al plate-PL2 & $T_{P L 2}(x)=\left(\frac{q_{a 2}}{\lambda_{P L} W_{P L}}-\frac{\dot{q}_{i}}{\lambda_{P L} W_{P L}}\right) \frac{x^{2}}{2}+C_{9} x+C_{10}$ \\
Insulation-INS & $T_{I N S}(y)=-\left(\frac{\dot{q}_{o}}{\lambda_{I N S}}\right) y+T_{0}$ \\
Plastic wall-W & $T_{W}(y)=\left(\frac{\left(\frac{\dot{q}_{i}}{\lambda_{W}}\right) y+T_{i}-\left(\frac{\dot{q}_{i}}{\lambda_{W}}\right) * H}{}\right.$ \\
\hline
\end{tabular}

\section{Supplementary materials}

Supplementary material associated with this article can be found, in the online version, at doi:10.1016/j.jirefrig.2018.01.012.

\section{References}

ANSYS, Inc: Ansys Fluent Theory Guide, Release 17.2. ANSYS, Inc., 2016.

ANSYS, Inc: Ansys Fluent Meshing User's Guide, Release 17.2. ANSYS, Inc., 2016.

Bansal, P.K., Chin, T.C., 2002. Design and modelling of hot-wall condensers in domestic refrigerators. Appl. Therm. Eng. 22, 1601-1617.

Bergman, T.L., Lavine, A.S., Incropera, F.P., DeWitt, D.P., 2011. Fundamentals of Heat and Mass Transfer, seventh ed. John Wiley \& Sons.

Colombo, E.G., Espindola, R.S., Knabben, F.T., Melo, C., 2016. A numerical and experimental study on skin condensers applied to household refrigerators. In: International Refrigeration and Air Conditioning Conference.

Gupta, J.K., Gopal, M.R., 2008. Modeling of hot-wall condensers for domestic refrigerators. Int. J. Refrig. 31, 979-988.

Hermes, C.J.L., Melo, C., Negrao, C.O.R., 2008. A numerical simulation model for plate-type, roll-bond evaporators. Int. J. Refrig. 31, 335-347.

Laguerre, O., Flick, D., 2004. Heat transfer by natural convection in domestic refrigerators. J. Food Eng. 62, 79-88.

Lee, T.H., Lee, J.S., Oh, S.Y., Lee, M.Y., Lee, K.S., 2002. Comparison of airside heat transfer coefficients of several types of evaporators of household freezer/refrigerators. In: International Refrigeration and Air Conditioning Conference.

Manneville, P., 2006. Rayleigh-Benard convection, thirty years of experimental theoretical, and modeling work, Dynamics of Spatio-Temporal Cellular Structures. Springer Tracts in Modern Physics, vol. 207. Springer, New York.

McGee, G.R., Schankula, M.H., Yovanovich, M.M., 1985. Thermal resistance of cylinder-flat contacts: Theoretical analysis and experimental verification of a linecontact model. Nucl. Eng. Des. 86, 369-381.

Rebora, A., Tagliafico, L.A., 1997. Thermal performance analysis for hot-wall condenser and evaporator configurations in refrigeration appliances. Int. J. Refrig. 21, 490-502. 